\title{
On the Formation of Hydrogen Emission Lines in Miras
}

\author{
Patrick de Laverny \\ Uppsala Astronomical Observatory, Box 515, S-75120 Uppsala, Sweden. \\ GRAAL, Université Montpellier II, France.
}

Christian Magnan

GRAAL, cc72, Université Montpellier II, 34000 Montpellier, France. Collège de France.

\begin{abstract}
We investigate non-LTE effects in the hydrogen recombination zone of Long-Period Variables of Mira type. We propose that the Balmer hydrogen emission lines could be formed in this deep layer. We show how the spectral cyclic variations of Miras could be mimicked with this interpretation. As a result the radius of a Mira in the Balmer transitions could be much smaller than its radius in the molecular absorptions, which are formed in higher layers.
\end{abstract}

\section{Introduction}

Long-Period variables of Mira type are giant pulsating AGB stars. Their spectrum exhibits large molecular absorptions typical of cool spectra. Balmer emission lines are also observed around the maximum luminosity of these stars. Qn the other hand, equilibrium departures are very important quite deep in Miras. One indeed observes shock-waves and large mass-loss rates. It is then very difficult to model these complex pulsating stars. However, the hydrodynamic effects have been investigated in some atmospheric models (see Bowen, 1988 for instance) and in some inner structure models (Tuchman et al., 1978 and Tuchman 1995). The inner structure models indicate that the hydrogen recombination zone (or $\mathrm{H}$ II/H I transition) is formed very high in a Mira $\left(R_{\mathrm{HII} / \mathrm{HI}} \approx 80-90 \% R_{\mathrm{Mira}}\right)$. Its density is also too low in order to assume LTE $\left(N_{\mathrm{HII} / \mathrm{HI}}<10^{16} \mathrm{~cm}^{-3}\right)$. The aim of this work is to estimate these non-LTE effects found in layers as deep as the hydrogen recombination zone.

\section{Simulation of the spectrum of a Mira}

We determine the spectrum emerging from an optically thick hydrogen envelope where non-LTE effects are taken into account. The physical properties of this envelope are close to those of the $\mathrm{H} \mathrm{II} / \mathrm{H} \mathrm{I}$ transition of a Mira (quite low density and large radius). We then show that emission lines are produced by recombination mechanisms in this transition zone (Magnan \& de Laverny, 1994 
and de Laverny \& Magnan, 1995). The profiles of these lines also exhibit a large central reversal.

Since the H II/H I transition of a Mira is surrounded by an extended cool atmosphere we have then to verify that the emission lines can emerge from the star. We have thus estimated the atmospheric absorption. As a result if the hydrogen recombination zone is formed high enough in the star the emerging spectrum is close to the radiation observed near the maximum luminosity of a Mira (Balmer emission lines are superposed on a very cool continuum).

It is also possible to mimic the cyclic spectral variations of a Mira (reddening of the continua, appearance/disappearance of the Balmer lines) with this crude model. It has been shown that the hydrogen recombination zone is shifted towards the centre of the star around the minimum luminosity of a Mira. Its radius can vary by $20 \%$ during the cycle (Tuchman et al., 1978). We have shown that such a contraction of the $\mathrm{H} \mathrm{II/H} \mathrm{I} \mathrm{transition} \mathrm{leads} \mathrm{to} \mathrm{larger} \mathrm{atmospheric}$ absorption. The emerging spectrum is then cooler without any Balmer emission lines. We thus mimic the spectrum of a Mira near its minimum luminosity.

\section{Conclusion}

We have proposed that the spectrum of a Mira could be composed of hydrogen recombination lines formed in a deep layer and cold absorption formed above (see for more details, Magnan \& de Laverny, 1995).

The cycle of a Mira can also be mimicked with this schematic model. At the maximum of luminosity, the hydrogen recombination zone is formed very high in the star. Balmer lines with a central reversal are formed by fluorescence mechanisms in this transition and emerge in emission from the atmosphere. On another hand the $\mathrm{H} \mathrm{II} / \mathrm{H}$ I transition is formed deeper in the star at its minimum luminosity. The Balmer lines disappear and the star is cooler.

Finally we would like to point out a crucial observational test to this interpretation. Since hydrogen lines are formed deeper in the star than the molecular absorption the radius of a Mira could vary strongly with wavelength. We then propose that its radius in the Balmer transitions is much smaller than its radius in the molecular absorption. Future interferometric measurements of Miras diameters in narrow filters could give interesting constraints to this model.

Acknowledgments. This work has been partly supported by a grant from the European Space Agency. We thank the IAU and French GdR "Milieux Circumstellaires" for their financial support to attend this colloquium.

\section{References}

Bowen, G.H. 1988, ApJ, 329, 299

de Laverny, P., \& Magnan, C. 1995, A\&A, submitted

Magnan, C., \& de Laverny, P. 1994, Astrofizika, Vol.37

Magnan, C., \& de Laverny, P. 1995, MNRAS, submitted

Tuchman, Y., Sack, N., \& Barkat, Z. 1978, ApJ, 219, 183

Tuchman, Y. 1995, this volume 